\title{
Photoelectrochemical studies on colloidal copper (I) oxide/modified with some organic semiconductors: Incentive for use of nanoparticle systems
}

\author{
KASEM K KASEM* and CARMEN DAVIS \\ National Information Mathematical Sciences Department, School of Arts and Science, Indiana University Kokomo, \\ Kokomo, IN 46904, USA
}

MS received 4 April 2008

\begin{abstract}
Colloidal $\mathrm{Cu}_{2} \mathrm{O}$ solutions were used to explore photonic activities at the semiconductor/electrolyte interface. Fluorescence spectroscopic studies were performed on $\mathrm{Cu}_{2} \mathrm{O}$ colloidal particles modified with some conjugated organic monomers such as 2-amino-phenyl pyrrole (2-APPy), tri-phenyl amine (TPA), or 2-thionyl pyrrole (2-Th-Py) to investigate the quantum absorbance efficiency at this inorganic/organic interface (IOI). Our study shows that colloidal $p$-type $\mathrm{Cu}_{2} \mathrm{O}$ possesses a bandgap with direct transition of $\approx 2 \cdot 2 \mathrm{eV}$ and indirect transition of $1.85 \mathrm{eV}$. The recorded rates of charge injection into colloidal $\mathrm{Cu}_{2} \mathrm{O}, \boldsymbol{k}_{\mathrm{ct}}$, were $2.31 \times 10^{9} \mathrm{~s}^{-1}, 5.05 \times$ $10^{8} \mathrm{~s}^{-1}$, and 7.22 $\times 10^{8} \mathrm{~s}^{-1}$ for 2-APPy, TPA and 2-Th-Py, respectively. The studied systems show more stability in colloidal form than in thin solid form. Results were interpreted using the optical and electrical parameters of the organic monomer such as ionization potential (IP), electron affinity (EA) and energy bandgap (Eg), and the barrier height at the IOI interface. Stability of the colloidal system is attributed to the physical dimensions of the photoactive system. The nano-colloidal particle offers a condition where its size is less than $\sqrt{ } D t$.
\end{abstract}

Keywords. Photoelectrochemistry; nano-particles; semiconductors; interface; colloidal.

\section{Introduction}

The great demand for renewable sources of energy has increased interest in the use of semiconductors in capturing solar radiation. The fact that some metal oxides possess semiconductor characteristics with bandgaps matching visible solar radiation adds great advantages to these inexpensive metal oxides over other more expensive semiconductors. The conversion of solar radiation into a controllable form can be more efficient if we focus on the range of the radiation spectrum with a relative high photon flux. Wavelengths within the range between 450 and $800 \mathrm{~nm}$ fit these criteria. Semiconductors with bandgaps between $3.1 \mathrm{eV}$ and $1.5 \mathrm{eV}$ can be used to capture and convert these radiations into chemical or photo-chemical energy.

A tangible effort is being made to develop a number of semiconductors in the form of thin film-electrodes or colloidal systems which might be used in solid or liquid photovoltaic cells (Spanhel et al 1987; Kamat and Dimitrijevic 1989; Gruszecki and Holmstrom 1998; Kumar et al 1998; Willner et al 1998; Cahen et al 2000; Shipway et al 2000; Kamat 2002; Adams et al 2003; George Thomas and Kamat 2003). Several methods were used to prepare

\footnotetext{
*Author for correspondence (kkasem@iuk.edu)
}

these semiconductors as single crystals or as polycrystalline materials. Examples of these methods are chemical vapour deposition and vacuum evaporation. In most of these methods, one material with a specific bandgap is being produced. Some studies were performed on metal chalcogenides such as sulfides, selenides, tellurides, but low conversion efficiencies were reported (Goidas et al 1990; Vogel et al 1990, 1994; Kohtani et al 1993; Liu and Kamat 1993; Graetzel 1997, 2001; Plass et al 2002; Peter et al 2003). Ordered assemblies of narrow bandgap semiconductor nanostructures, if employed, can be convenient systems to harvest visible light energy. The photocurrent obtained using such nanoparticle assemblies is often low, as fast charge recombination limits photocurrent generation. By employing composite semiconductors, however, it has been possible to improve the efficiency of charge separation through charge rectification by modifying the surface of the base semiconductor with either inorganic or organic semiconductors. As thin films of $\mathrm{Cu}_{2} \mathrm{O}$ show poor resistance to photo corrosion (Nagasubramanian et al 1981; Hara et al 1998a), an alternative form of $\mathrm{Cu}_{2} \mathrm{O}$ may show a greater stability than that of the thin solid film.

In this paper, we investigate the photonic behaviour of diverse sizes of $\mathrm{Cu}_{2} \mathrm{O}$ colloidal nanoparticles with special emphasis on the effectiveness in capturing solar radiation. Furthermore, because of the great surface area that a colloidal solution can offer, adsorption of organic semi- 
conductor material is very likely and inorganic/organic interface (IOI) assemblies can be produced. Three conjugated organic compounds were chosen, 2-APPy, TPA, 2-Th-Py. We investigate the effect of these organic semiconductors on the photonic behaviour of these assemblies and judge how much of solar radiation can be harvested.

\section{Experimental}

\subsection{Reagents}

All reagents were of analytical grade. All solutions were prepared using deionized water, unless otherwise stated.

\subsection{Instrumentation}

All electrochemical experiments were carried out using a conventional three-electrode cell consisting of $\mathrm{Pt}$ wire as a counter electrode, $\mathrm{Ag} / \mathrm{AgCl}$ as a reference electrode and BAS $100 \mathrm{~W}$ electrochemical analyser (Bioanalytical Co.) was used to perform the electrochemical and impedance studies. X-ray photoelectron spectroscopy (XPS) was performed using a Perkin Elmer 5500 ESCA. An Amery $1000 \mathrm{~A}$ was used to produce scanning electron micrographs of the polymer films. Both UV-VIS spectrophotometer and reflectance UV-VIS were used to generate absorption spectra of the thin solid films on ITO electrodes or for the colloidal oxide. An Olympus BX-FLA60 reflected light fluorescence microscope working with polarized light at a range between $\lambda 330$ and $550 \mathrm{~nm}$ was used to determine the particle size of colloidal particles.

Fluorescence lifetime measurements were recorded using a Chronos (ISS, Champaign, IL) with a laser diode as the excitation source (Tatarets et al 2006).

\subsection{Synthesis of colloidal $\mathrm{Cu}_{2} \mathrm{O}$}

Colloidal $p$-type $\mathrm{Cu}_{2} \mathrm{O}$ was generated using a hydrolysis method. In this method, a buffered basic solution of copper acetate in the presence of hydrazine or with Fehling reagent, was heated to $90^{\circ} \mathrm{C}$ under constant stirring for $2 \mathrm{~h}$. In all cases, colloidal oxide was allowed to stile, then filtered, rinsed and dried at $200^{\circ} \mathrm{C}$ for $10 \mathrm{~h}$. The colloidal particles were further dispersed into water using high energy sonicators.

\section{Results and discussion}

\subsection{Redox potential of organic semiconductors}

Cyclic voltammetric studies were carried out, unless otherwise stated, in acetonitrile solutions for the 2-APPy, 2-Th$\mathrm{Py}$, and TPA. Figure 1 displays the $\mathrm{CV}$ for the polymer film of each of the studied monomers. The CVs of the monomers were not different from those of the polymer films. The onset oxidation potential corresponding to each of these molecules are recorded in table 1 .

\subsection{Characterization of $\mathrm{Cu}_{2} \mathrm{O}$ nanoparticles}

Figure 2 illustrates the absorption spectra of meso-size particles of $\mathrm{Cu}_{2} \mathrm{O}$. In large particles direct and indirect transitions are possible. We used the following equations (Robert et al 1998) to calculate the direct and indirect transition band structures for $\mathrm{Cu}_{2} \mathrm{O}$ spectra displayed in figure 2 .

$$
\begin{aligned}
& \left(\alpha E_{\gamma}\right)^{2} \propto E_{\gamma}-E_{\mathrm{gd}}, \\
& \alpha \propto \frac{\left(E_{\gamma}+E_{\mathrm{p}}-E_{\mathrm{gi}}\right)^{2}}{e^{(E \gamma / k T)}-1}+\frac{\left(E_{\gamma}-E_{\mathrm{p}}-E_{\mathrm{gi}}\right)^{2} e^{(E \gamma / k T)}}{e^{(E \gamma / k T)}-1},
\end{aligned}
$$

where $\alpha$ is absorption coefficient, $E_{\mathrm{g}}$ the optical bandgap. Figures 3A and B display Tauc's plots from which we obtained the direct and indirect bandgap transitions

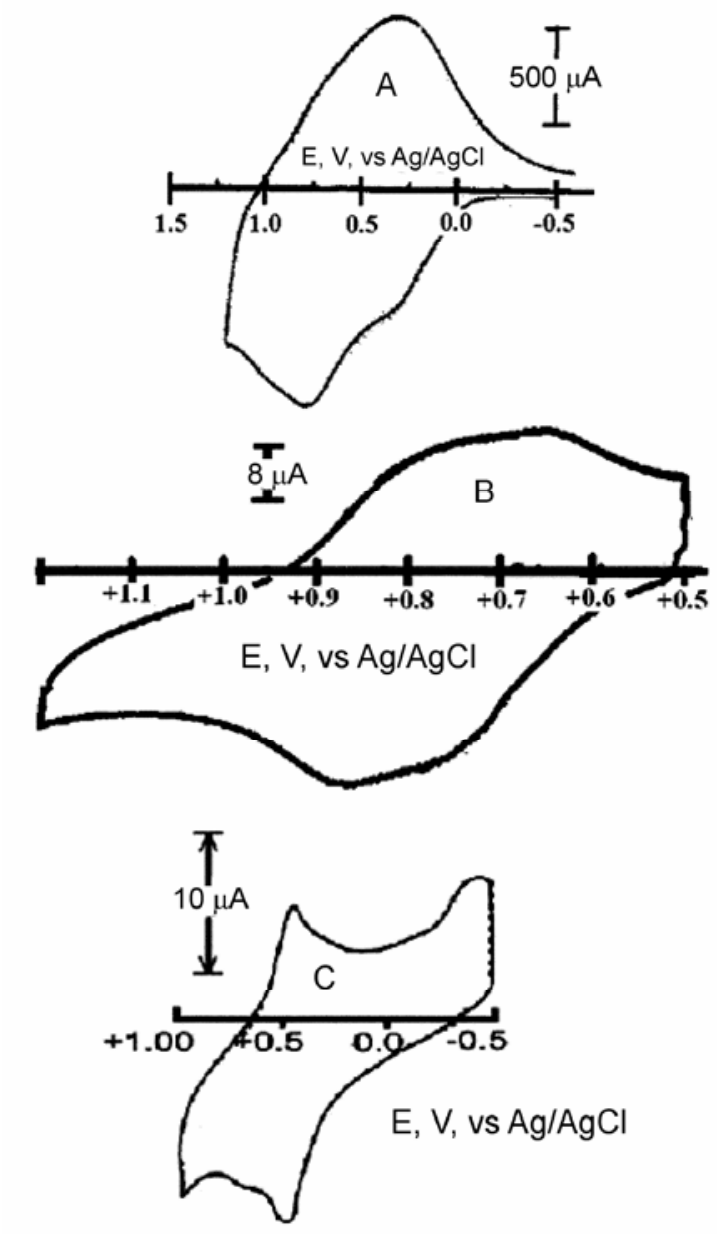

Figure 1. CV in acetonitrile perchlorate electrolyte of Pt electrode modified with A. poly 2-Th-Py, B. poly TPA, and C. poly 2-APPy (protonated in aqueous $\mathrm{HClO}_{4}$ ). Scan rate $(100 \mathrm{mV} / \mathrm{s})$. 
shown in the inset diagram of figure 3. This diagram indicates that colloidal $\mathrm{Cu}_{2} \mathrm{O}$ possesses bandgaps with a direct transition of $\approx 2 \mathrm{eV}$ and an indirect transition of $1.85 \mathrm{eV}$. The nano particles of $\mathrm{Cu}_{2} \mathrm{O}$ show greater bandgap than larger particles $(500-1000 \mathrm{~nm})$. The calculated optical bandgap for nano-crystals of $\mathrm{Cu}_{2} \mathrm{O}$ (data not displayed) is $2.52 \mathrm{eV}$, a value that is consistent with nano or quantum dot size particles.

\subsection{Photo-absorption activities of IOI}

Fluorescence emission studies were performed on a copper (I) oxide colloid modified with 2-APPy, 2-Th-Py, and TPA. To detect the absorption of the emitted photon from organic semiconductors, a reference system consisting of colloidal $\mathrm{SiO}_{2}(E g>5 \mathrm{eV})$ and an organic semiconductor was studied before and after doping with $\mathrm{Cu}_{2} \mathrm{O}$. In the

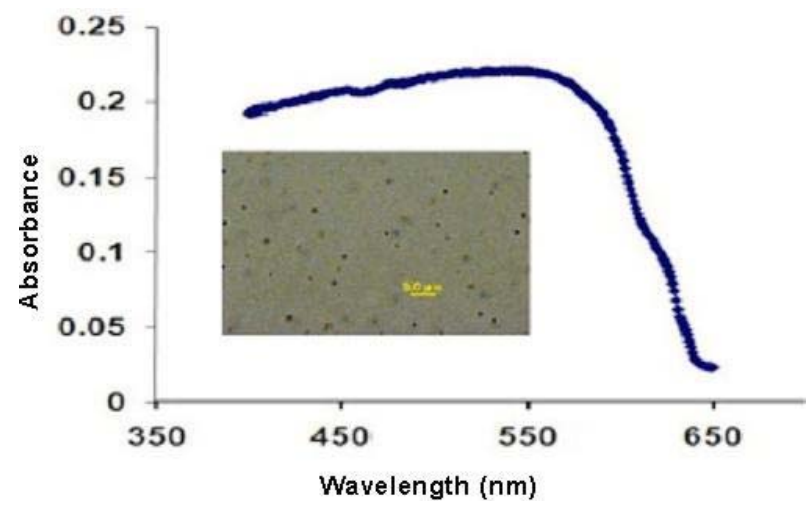

Figure 2. Absorption spectra of meso-sized particles of $\mathrm{Cu}_{2} \mathrm{O}$. Inset: the fluorescence-microscopic image of the particles.
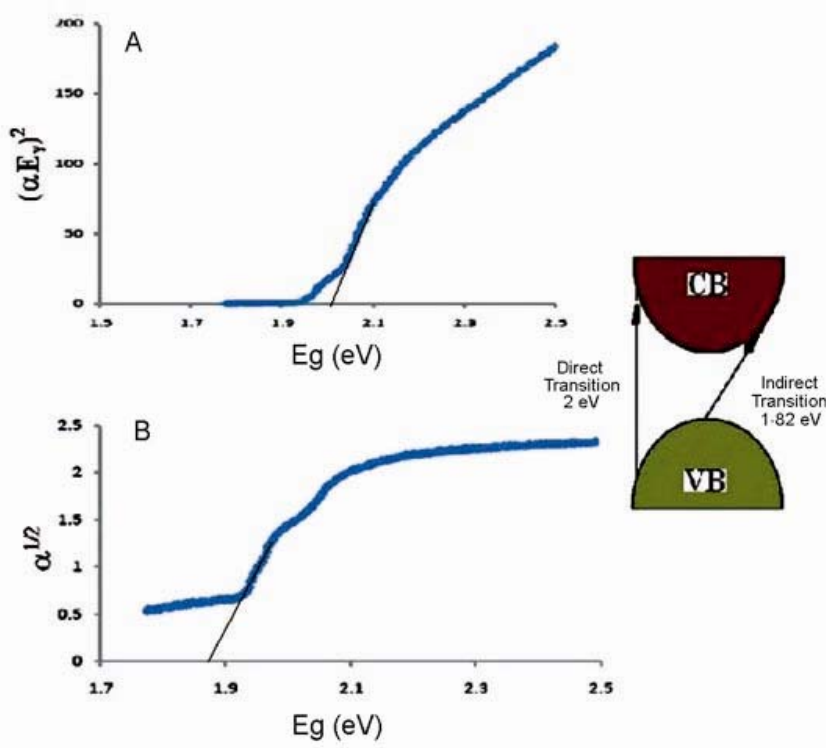

Figure 3. A. Direct and $\mathbf{B}$. indirect bandgap meso-size particles of $\mathrm{Cu}_{2} \mathrm{O}$ (Inset: bandgap diagram) absence of $\mathrm{Cu}_{2} \mathrm{O}$, all fluorescence emissions were detected, as no injection will take place into $\mathrm{SiO}_{2}$ particles due to the very large bandgap and band positions with HUMO and LOMO in the organic semiconductors. In the presence of $\mathrm{Cu}_{2} \mathrm{O}$, less fluorescence emission was recorded as $\mathrm{Cu}_{2} \mathrm{O}$ absorbed some of these emitted photons. Figure 4 displays the fluorescence emission spectra of the colloidal system consisting of $\mathrm{SiO}_{2}$ and 2-APPy (figure 4A), 2-Th-Py (figure $4 \mathrm{~B}$ ), and TPA (figure 4C) before and after doping with $\mathrm{Cu}_{2} \mathrm{O}$.

Understanding the photonic activities at the studied IOI shown in figure 4 requires information about the characteristic energies at IOI such as EA, IP, and $E g$ of the organic semiconductor and $\varphi$ (barrier potential) at IOI
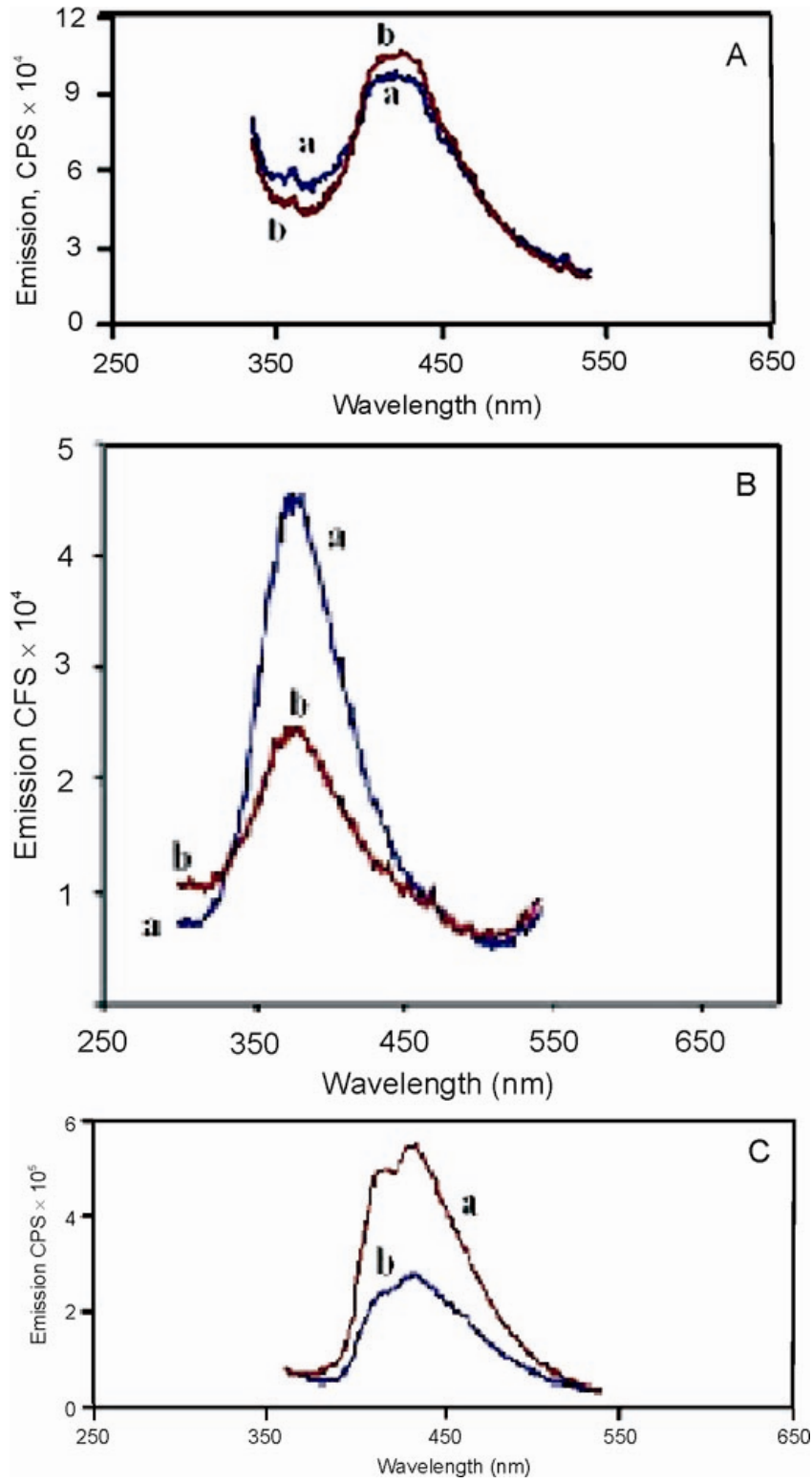

Figure 4. Fluorescence emission on a. $\mathrm{SiO}_{2}$. b. $\mathrm{SiO}_{2}$ doped with $\mathrm{Cu}_{2} \mathrm{O}$ (excitation $\lambda=320 \mathrm{~nm}$; A. 2-APPy; B. 2-Th-Py and C. TPA). 
Table 1. Chemical and photo-electrochemical data of IOI components.

\begin{tabular}{|c|c|c|c|}
\hline Property & 2-APPy & TPA* & 2-Th-Py \\
\hline Onset oxidation potential (vs $\mathrm{Ag} / \mathrm{AgCl}$ ) & $0 \cdot 3 \mathrm{~V}$ & $0 \cdot 8 \mathrm{~V}$ & $0 \cdot 4$ \\
\hline IP (ionization potential $(\mathrm{eV})$ ) & 4.7 & $5 \cdot 2$ & $4 \cdot 8$ \\
\hline Eg (Bandgap, eV) & $\approx 2 \cdot 8$ & $\approx 2 \cdot 2-2 \cdot 4$ & $\approx 1.8$ \\
\hline EA (electron affinity, eV) & 1.9 & 3.0 to $2 \cdot 8$ & $3 \cdot 0$ \\
\hline Barrier height $\varphi(\mathrm{eV})$ at $\mathrm{Cu}_{2} \mathrm{O} /$ molecule interface & $3 \cdot 0$ & 1.9 to $2 \cdot 1$ & 1.9 \\
\hline Life time (ns) & 0.437 & 1.98 & 1.4 \\
\hline$\%$ of Fluoresces emission absorbed by $\mathrm{Cu}_{2} \mathrm{O}$ & 0 & $72 \cdot 7$ & $65 \cdot 0$ \\
\hline
\end{tabular}

*Two-photon emissions

interface. These quantities constitute the potential map of the IOI assemblies.

The potential maps of the studied organic semiconductors were calculated using the following relations:

$$
\mathrm{IP}=E_{\mathrm{ox}}=E_{\mathrm{ox}}^{\prime}+4 \cdot 4 \mathrm{eV},
$$

where $E_{\mathrm{ox}}^{\prime}$ is onset oxidation potential, IP the ionization potential, and EA the electron affinity.

$$
\mathrm{IP}=\mathrm{EA}+\mathrm{Eg} .
$$

Table 1 lists the data corresponding to the studied three organic compounds. These data give an insight to the potential map of the studied IOI assemblies.

The data listed in table 1 shows that the IOI made of $\mathrm{Cu}_{2} \mathrm{O}$ /organic semiconductor absorbs the least amount of emitted fluorescence photons with 2-APPy adsorbent. The percentages of absorbed photons at the IOI assemblies were $0 \%, 65 \%$, and $72 \%$ for 2-APPy, 2-Th-Py and TPA, respectively. The zero absorption percentage at $\mathrm{Cu}_{2} \mathrm{O} / 2$-APPy can be attributed to the fact that 2-APPy is a $p$-type organic semiconductor as indicated by its low EA $(1.9 \mathrm{eV})$, and $\mathrm{Cu}_{2} \mathrm{O}$ is also $p$-type. The IOI favours charge separation and further injection when the condition for $p-n$ junction is fulfilled. That is not the case with $\mathrm{Cu}_{2} \mathrm{O} / 2$-APPy interface. The barrier height (barrier height, $\varphi=$ work function of $\mathrm{Cu}_{2} \mathrm{O}-\mathrm{EA}$ of 2-APPy) is $3 \mathrm{eV}$. This value is high enough to make charge injection thermodynamically unfavourable. The greater absorption percentage recorded for TPA and 2-Th-Py can be explained by the fact that TPA and 2-Th-Py are $n$-type hole-transporting materials due to their higher EA (table 1), and thus has a lower barrier potential. It is worthwhile noticing that the physical quantities recorded in table 1 , other than $\varphi$, assume symmetric conditions for each individual organic molecule. In reality IOI interface, symmetry of the adsorbed organic molecule is broken. This will cause shifts in some molecular energy of the adsorbed molecule in comparison with unadsorbed ones. As a result of this asymmetric status of the adsorbed organic molecules, the energy barrier for charge transfer is rearranged.

Table 1 also shows that the IOI with TPA has a greater absorption than that with 2-Th-Py. Furthermore, the measured life time of the excited state for TPA $(1.98 \mathrm{~ns})$ is longer than that of 2-Th-Py (1.4 ns). Both the structure and the measured life time indicate that TPA offers larger surface area of delocalization of the formed radical cation. This allows for more stable intermediates with different energy states that favour charge injection into $\mathrm{Cu}_{2} \mathrm{O}$ particles at the IOI assembly.

An important factor that may influence the photonic activities at these IOIs is the relative life times of the excited states created after illumination. The determined fluorescence life time of 2-APPy, TPA and 2-Th-PY are $0.437 \mathrm{~ns}, 1.98 \mathrm{~ns}$ and $1.4 \mathrm{~ns}$, respectively. The shorter life time of the organic semiconductor compared to that reported for $\mathrm{Cu}_{2} \mathrm{O}$ suggests that organic semiconductors will inject charges into the copper (I) oxide colloidal particles, because the hole in the valence band has longer life than the excitons generated from 2-APPy or TPA, or 2Th-Py. For illuminated $\mathrm{Cu}_{2} \mathrm{O}$, there are three possible ways for the generated electron-hole pairs to be consumed; first is the recombination of electron-hole, second is electron transfer to another chemical agent, and the third is the reduction of $\mathrm{Cu}^{+}$ions to metallic copper. The shorter lifetime of organic semiconductors excitons compared with $\mathrm{Cu}_{2} \mathrm{O}$ suggests that these electrons can be injected to neutralize the holes in $\mathrm{VB}$ of $\mathrm{Cu}_{2} \mathrm{O}$. This will certainly reduce the electron-hole recombination process. The lifetime of the polaron shown in figure 4 determines whether these polarons will be partially or totally consumed in an oxidation of a chemical agent in conjunction with this illuminated IOI.

\subsection{Stability of colloidal $\mathrm{Cu}_{2} \mathrm{O}$ /organic interface}

The IOI were used several times which generated the same results. This indicates its stability towards photocorrosion. Colloidal nano-particles of $\mathrm{Cu}_{2} \mathrm{O}$ show a great stability against illumination for almost $1900 \mathrm{~h}$ (Hara et al 1998b). A thin solid film of $\mathrm{Cu}_{2} \mathrm{O}$ prepared electrochemically using cycling the potential of pure copper sheet between $-0.6 \mathrm{~V}$ and $+0.1 \mathrm{~V}$ vs $\mathrm{Ag} / \mathrm{AgCl}$ electrode (figure 5A and inset) shows a very poor stability upon illuminations as shown in figure 5B. The greater stability of nano particles of $\mathrm{Cu}_{2} \mathrm{O}$ than that of thin solid film of $\mathrm{Cu}_{2} \mathrm{O}$ can be attributed to two factors: (I) Morphological factor: We believe that photo corrosion is mostly due to the galvanic action that takes place following the forma- 
tion of excited state. If the dimension of the $\mathrm{Cu}_{2} \mathrm{O}$ cluster is greater than $\sqrt{ } D t$, (where $D$ is the diffusion coefficient of $\mathrm{Cu}+$ ions in the cluster, and $t$ the excited state life time in $\mathrm{s}$ ), the galvanic cell will be formed and degradation of $\mathrm{Cu}_{2} \mathrm{O}$ takes place. As the thin solid film offers continuous, polycrystalline structure with larger area which is greater than $\sqrt{ } D t$, such phenomena will not take place in nano structure. $\mathrm{Cu}_{2} \mathrm{O}$ nanoparticles $(50 \mathrm{~nm}$ or less) have more resistance to photo corrosion than particles of mesoor micro-sizes. Certainly $\mathrm{Cu}_{2} \mathrm{O}$ quantum dot will have the greatest stability against photo-corrosion and (II) structural factor in which the nano structure offers larger bandgap than that offered in condensed thin solid film. This larger bandgap offers energy map in which a VB and $\mathrm{CB}$ energy shifts to energy edge where $\mathrm{E} \mathrm{OH}^{-} / \mathrm{H}_{2} \mathrm{O}$ is less than the $\mathrm{E} p d$ (free energy of oxidation of the $\mathrm{Cu}_{2} \mathrm{O}$ ), and $\mathrm{E} \mathrm{H}^{+} / \mathrm{H}_{2} \mathrm{O}$ is greater than the $\mathrm{E}$ nd (free energy of reduction of $\mathrm{Cu}_{2} \mathrm{O}$ ).

\section{Conclusions}

The use of organic semiconductors in conjunction with colloidal inorganic semiconductors will increase the process of light harvesting, suppress electron-hole recombination rate, and increases the quantum conversion efficiency of the IOI system. Unlike chemical doping of inorganic semiconductors, modification of the surfaces with organic semiconductors will expand the photonabsorption range and enhance the charge separation and injection as well. Our results give rise to more incentive of using colloidal metal oxide semiconductors in IOI assem-
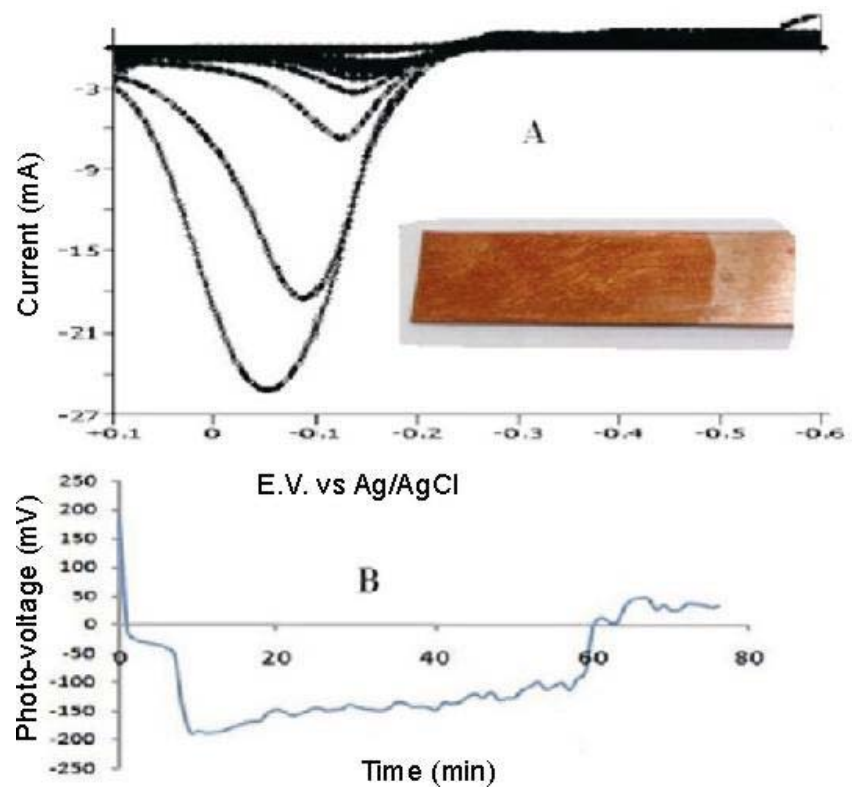

Figure 5. A. Electrodeposition of $\mathrm{Cu}_{2} \mathrm{O}$ film from $0 \cdot 1 \mathrm{M} \mathrm{LiOH}$ aqueous electrolyte (Inset: film image of $\mathrm{Cu}$ specimen) and $\mathbf{B}$. photo-voltage time curve for thin $\mathrm{Cu}_{2} \mathrm{O}$ film shown in $\mathrm{A}$, in aqueous $\mathrm{KCl}$ electrolyte under illumination. blies due to the great stability and larger surface area that they can offer. The great absorption of photons generated by excitation using short UV radiation suggests that TPA or 2-Th-Py can be used as a major component in high UV radiation protection paint.

\section{Acknowledgements}

This work was supported by Indiana University Summer faculty fellowship program.

\section{References}

Adams D et al 2003 J. Phys. Chem. B107 6668

Cahen D, Hodes G, Graetzel M, Guillemoles J F and Riess I 2000 J. Phys. Chem. 1042053

George Thomas K and Kamat P V 2003 Acc. Chem. Res. 36888

Goidas K R, Bohorques Maraia and Kamat P V 1990 J. Phys. Chem. B94 6435

Graetzel M 1997 Nanocrystalline electronic junctions. in Semiconductor nanoclusters-Physical, chemical and catalytic aspects (eds) P V Kamat and D Meisel (Amsterdam: Elsevier Science) pp 353-375

Graetzel M 2001 Nature 414338

Gruszecki T and Holmstrom B J 1998 Sol. Energy Mater., Sol. Cells 51243

Hara Michikazu, Kondo Takeshi, Komoda Mutsuko, Ikeda Sigeru, Shinohara Kiyoaki, Tanaka Akira, Kondoa Junko N and Domen Kazunari 1998a Chem. Commun. 357

Hara Michikazu, Kondo Takeshi, Komoda Mutsuko, Ikeda Sigeru, Kondo Junko N, Domen Kazunari, Shinohara Kiyoaki and Tanaka Akira 1998b Chem. Commun. 357

Kamat P V 2002 J. Phys. Chem. B106 7729

Kamat P V and Dimitrijevic N M 1989 J. Phys. Chem. 934259

Kohtani S, Kudo A and Sakata T 1993 Chem. Phys. Lett. 206 166

Kumar M, Sharan K and Sharon M J 1998 Sol. Energy Mater., Sol. Cells 5135

Liu D and Kamat P V 1993 J. Phys. Chem. 9710769

Nagasubramanian G, Giods A S and Bard A J 1981 J. Electrochem. Soc. 1282158

Peter L M, Wijayantha K G U, Riley D J and Waggett J P 2003 J. Phys. Chem. B107 8378

Plass R, Pelet S, Krueger J, Graetzel M and Bach U 2002 J. Phys. Chem. B106 7578

Robert A, Leeuwen van, Hung Chen-jen, Kammler Danlel R and Switzer Jay A 1998 J. Phys. Chem. 9915247

Shipway A N, Katz E and Willner I 2000 Chem. Phys. Chem. 1 18

Spanhel L, Weller H and Henglein A 1987 J. Am. Chem. Soc. 109

Tatarets Anatoliy L, Fedyunyayeva Irina A, Dyubko Tatyana S, Povrozin Yevgeniy A, Doroshenko Andrey O, Terpetschnig Edward A and Patsenker Leonid D 2006 Anal. Chim. Acta 570214

Vogel R, Pohl K and Weller H 1990 Chem. Phys. Lett. 174 241

Vogel R, Hoyer P and Weller H 1994 J. Phys. Chem. 983183

Willner I, Kaganer E, Joselevich E, Durr H, David E, Gunter M J and Johnston M R 1998 Coord. Chem. Rev. 171261 